\title{
Preconditioning with ethyl 3,4-dihydroxybenzoate augments aerobic respiration in rat skeletal muscle
}

\author{
Charu Nimker \\ Deependra Pratap Singh \\ Deepika Saraswat \\ Anju Bansal
}

Experimental Biology Division, Defence Institute of Physiology and Allied Sciences, Defense Research and Development Organisation, Timarpur, Delhi, India

Correspondence: Anju Bansal Experimental Biology Division, Defence Institute of Physiology and Allied Sciences, Defense Research and Development Organisation, Lucknow Road, Timarpur, Delhi II 0054, India

Email anjubansaldipas@gmail.com
This article was published in the following Dove Press journal:

Hypoxia

13 May 2016

Number of times this article has been viewed
Abstract: Muscle respiratory capacity decides the amount of exertion one's skeletal muscle can undergo, and endurance exercise is believed to increase it. There are also certain preconditioning methods by which muscle respiratory and exercise performance can be enhanced. In this study, preconditioning with ethyl 3,4-dihydroxybenzoate (EDHB), a prolyl hydroxylase domain enzyme inhibitor, has been investigated to determine its effect on aerobic metabolism and bioenergetics in skeletal muscle, thus facilitating boost in physical performance in a rat model. We observed that EDHB supplementation increases aerobic metabolism via upregulation of HIF-mediated GLUT1 and GLUT4, thus enhancing glucose uptake in muscles. There was also a twofold rise in the activity of enzymes of tricarboxylic acid (TCA) cycle and glycolysis, ie, hexokinase and phosphofructokinase. There was an increase in citrate synthase and succinate dehydrogenase activity, resulting in the rise in the levels of ATP due to enhanced Krebs cycle activity as substantiated by enhanced acetyl-CoA levels in EDHB-treated rats as compared to control group. Increased lactate dehydrogenase activity, reduced expression of monocarboxylate transporter 1, and increase in monocarboxylate transporter 4 suggest transport of lactate from muscle to blood. There was a concomitant decrease in plasma lactate, which might be due to enhanced transport of lactate from blood to the liver. This was further supported by the rise in liver pyruvate levels and liver glycogen levels in EDHB-supplemented rats as compared to control rats. These results suggest that EDHB supplementation leads to improved physical performance due to the escalation of aerobic respiration quotient, ie, enhanced muscle respiratory capacity. Keywords: cellular metabolism, exercise, ethyl 3,4-dihydroxybenzoate, prolyl hydroxylase enzyme

\section{Introduction}

Skeletal muscle is a highly adaptive tissue. It responds to environmental and physiological challenges and exhibits a remarkable ability to adapt to altered demands by changes in size, fiber type, and metabolism. ${ }^{1,2}$ Training adaptation primarily includes capillarization, altered glycolytic flux, and increased mitochondrial density. ${ }^{3,4}$ It also includes increased capacity for oxidative metabolism of fatty acids and carbohydrates and enzymes of citrate cycle. ${ }^{5}$ All these adaptations lead to increase in muscle respiratory capacity and thereby play a major role in improving physical performance. In the process, multiple biological events are involved, which are modulated by a number of genes along with various translational and posttranslational events. A single bout of exercise can elicit increase in transcription rate of various regulatory genes, including peroxisome proliferator-activated receptor $\gamma$ coactivator 1 (PGC-1) and mitochondrial transcription factor (TFAM), and metabolic genes, viz, carnitine 
palmitoyltransferase I (CPTI), pyruvate dehydrogenase kinase 4 (PDK4), mitochondrial uncoupling protein 3 (UCP-3), and hemoxygenase 1 (HO-1), which function mainly during the recovery period. ${ }^{6-8}$

Hypoxia is the primary stimuli to trigger muscle adaptation during exercise, which is governed by a transcription factor, hypoxia-inducible factor (HIF-1), a master regulator of hypoxia-inducible genes. ${ }^{9-11}$ Prolyl hydroxylase domain enzymes degrade HIF-1 $\alpha$ under normoxia, whereas under hypoxia, HIF is stabilized due to inactivation of these enzymes. Thus, stabilization of HIF-1 $\alpha$ by prolyl hydroxylase domain enzyme inhibition might be used as a potential strategy to modulate metabolic adaptation in skeletal muscle, resulting in enhanced physical performance.

Earlier studies on ethyl 3,4-dihydroxybenzoate (EDHB), from our laboratory, have reported the antioxidant effect of EDHB in rat L6 cells under hypoxia, improvement in physical performance, and its protective effect against exerciseinduced damage in rats. ${ }^{12,13}$ Recently, we also studied its role in attenuation of hypobaric hypoxia-mediated vascular leakage in the brain. ${ }^{14}$ The aim of this study was to investigate EDHB-derived skeletal muscle adaptation to exercise by enhancing various metabolic responses, thus exploiting the potential of EDHB as a preconditioning agent for endurance stress in the skeletal muscles.

\section{Materials and methods Ethical approval}

All animal procedures were approved by the Institutional Animal Ethics Committee, Defence Institute of Physiology and Allied Sciences, and were in compliance with the Committee for the Purpose of Control and Supervision of Experiments on Animals, India. Efforts were made to minimize animal suffering and number of animals used for experimental purpose. Male Sprague Dawley rats (170 $\pm 10 \mathrm{~g})$ were maintained in the institute's animal house facility in a controlled environment $\left(24^{\circ} \mathrm{C} \pm 2^{\circ} \mathrm{C}\right)$ with 12 -hour light/dark cycle, and relative humidity was maintained at $40 \%-50 \%$. Animals were fed standard pelletized diet (Lipton India Ltd. Vijaywada, India) and water ad libitum. Body weight and food and water intake were measured daily. Animals were maintained under the surveillance of a qualified veterinarian from the institute.

\section{Materials}

All chemicals were purchased from Sigma-Aldrich Co. (St Louis, MO, USA) and Sisco Research Laboratories Pvt. Ltd. (Mumbai, India). Antibodies were purchased from Santa Cruz Biotechnology Inc. (Dallas, TX, USA).
In our previous studies on dose response, we found that EDHB at the dose of $50 \mathrm{mg} / \mathrm{kg}$ body weight for 3 days significantly improved the running time till exhaustion in rats. Therefore, all the experiments were carried out at this concentration. $^{13}$

\section{Physical performance and tissue collection}

To test the physical performance of rats and how it is influenced by preconditioning with EDHB, four groups of randomly selected rats ( $\mathrm{n}=8$ /group) were studied: 1 ) control sedentary (CS), 2) EDHB-treated sedentary (DS), 3) control training (CT), and 4) EDHB-treated training (DT). Rats in treated groups were supplemented with $50 \mathrm{mg} / \mathrm{kg}$ body weight of EDHB (intraperitoneal), while control groups were supplemented with the same amount of vehicle. Rats were subjected to treadmill training (Columbus Instruments International Corporation, Columbus, OH, USA) as described earlier. ${ }^{13,15}$ On the eleventh day, running time till exhaustion was measured in a blinded fashion. Animals were considered exhausted when unable to run on the treadmill, despite in contact with the shock bar at the rear end of the treadmill. Animals were sacrificed with an overdose of ketamine chloral hydrate and xylazine. Blood was collected by renal portal vein, and blood parameters were estimated immediately. Remaining blood was centrifuged at $1,000 \mathrm{xg}$ for 20 minutes, and plasma was processed immediately or kept at $-80^{\circ} \mathrm{C}$ for further analysis. Whole gastrocnemius muscle was collected from the calf region of hind limbs of rats, and red gastrocnemius muscle was separated, washed with phosphate-buffered saline twice, snap frozen after removal of hair and fatty tissue, and stored at $-80^{\circ} \mathrm{C}$ for subsequent biochemical and protein expression studies. Red gastrocnemius muscle was chosen because it is actively recruited during exercise and is highly oxidative in nature. ${ }^{16}$

\section{Assessment of bioenergetics}

Activities of enzymes involved in cellular respiration in red gastrocnemius muscle were measured spectrophotometrically as a marker of aerobic vs anaerobic metabolism. The activity of citrate synthase (citrate synthase, EC 2.3.3.1) was determined by the method of Srere ${ }^{17}$ at $\lambda_{\text {max }}=412 \mathrm{~nm}$. Assay for succinate dehydrogenase (SDH, EC 1.3.99.1) was adopted from the method of Veeger et al. ${ }^{18}$ This enzyme must be assayed as soon as possible after cell disruption. The rate of decrease in absorbance was measured at $455 \mathrm{~nm}$. Hexokinase activity was determined by a coupled enzyme assay, in which glucose is converted to glucose-6-phosphate by hexokinase by the method of Holroyde and Trayer. ${ }^{19}$ The increase in absorbance 
at $340 \mathrm{~nm}$ was recorded at $30^{\circ} \mathrm{C}$. Phosphofructokinase (PFK, EC 2.7.1.11) activity in muscle was measured by the method of Ling et al. ${ }^{20}$ Change in optical density was measured at $340 \mathrm{~nm}$ at $28^{\circ} \mathrm{C}$. Lactate dehydrogenase (LDH; EC 1.1.1.2) activity in skeletal muscle homogenate was estimated using a commercial assay kit from Randox (Crumblin, UK), as per the manufacturer's instructions. The decrease in absorbance was measured at $340 \mathrm{~nm}$.

\section{Estimation of biochemical levels}

Pyruvate was estimated in muscle and liver, while acetylCoA and glycogen were estimated in muscle by a coupled enzyme assay colorimetrically at $(570 \mathrm{~nm}) /$ fluorometrically at $\left(\lambda_{\mathrm{ex}}=535 / \lambda_{\mathrm{em}}=587 \mathrm{~nm}\right)$ using a commercial assay kit from Sigma-Aldrich Co., as per the manufacturer's instructions. Lactate level was measured in blood plasma using a commercial assay kit from Randox, as per the manufacturer's instructions. The optical density of the purple product was estimated at $340 \mathrm{~nm}$ with the spectrophotometer. The concentration of inorganic phosphate $(\mathrm{Pi})$ increases during intense skeletal muscle activity mainly due to the breakdown of creatine phosphate. Pi was estimated by the method of Goldenberg and Fernandez. ${ }^{21}$ The developed color was measured at $660 \mathrm{~nm}$ after 20 minutes.

\section{ATP level}

ATP is the primary energy currency of living systems. Virtually, all energy-requiring processes utilize the chemical energy stored in the phosphate bond of ATP. ATP bioluminescent assay kit (Sigma-Aldrich Co.) was used for the quantitative bioluminescent determination of ATP. The amount of light produced was measured using a multimode reader (FLUOstar Omega; BMG Labtech, Ortenberg, Germany).

\section{Protein expression studies by Western blotting}

Muscle samples were homogenized in four volumes of icecold buffer A (0.5 M sucrose, $10 \mathrm{mM}$ 4-(2-hydroxyethyl)-1piperazineethanesulfonic acid (HEPES), pH 7.9, $10 \mathrm{mM} \mathrm{KCl}$, $1.5 \mathrm{mM} \mathrm{MgCl}_{2}, 10 \%$ glycerol, $1 \mathrm{mM}$ ethylenediaminetetraacetic acid [EDTA], $1 \mathrm{mM}$ dithiothreitol [DTT], and $1 \mathrm{mM}$ phenylmethanesulfonyl fluoride) fortified with protease inhibitor cocktail (Sigma-Aldrich Co.). The homogenate was kept on ice for 15 minutes, and Nonidet (NP 40) was added to make a final concentration of $0.6 \%$, followed by centrifugation at $2,000 \times g$ for 10 minutes at $4^{\circ} \mathrm{C}$. The supernatant (cytosolic fraction) was collected and stored at $-80^{\circ} \mathrm{C}$. The pellet was dissolved in one volume of ice-cold buffer B (20 mM HEPES, pH 7.9, $0.3 \mathrm{mM} \mathrm{NaCl}, 1.5 \mathrm{mM} \mathrm{MgCl}_{2}$,
20\% glycerol, $0.2 \mathrm{mM}$ EDTA, $0.5 \mathrm{mM}$ DTT, and $0.5 \mathrm{mM}$ phenylmethanesulfonyl fluoride) fortified with protease inhibitor cocktail. It was kept on ice for 30 minutes, followed by centrifugation at $20,000 \times g$ for 15 minutes at $4^{\circ} \mathrm{C}$. The supernatant containing the nuclear fraction was aliquoted and stored at $-80^{\circ} \mathrm{C}$. Protein concentrations were quantified by Lowry's method.

Protein expression of glucose transporters, GLUT1 and GLUT4, pyruvate dehydrogenase kinase (PDK1 and 4), CPTI, and lactate transporters (monocarboxylate transporter 1 [MCT1] and MCT4), was studied in the cytosolic extract of red gastrocnemius muscle. Briefly, muscle homogenate containing $50 \mu \mathrm{g}$ of protein was subjected to sodium dodecyl sulfatepolyacrylamide gel electrophoresis and electroblotted onto a nitrocellulose membrane (EMD Millipore, Billerica, MA, USA). The membranes were blocked with $3 \%$ bovine serum albumin for 2 hours, washed with Tris-buffered saline containing Tween 20 (TBST, 0.1\%), and probed with respective mouse/rabbit monoclonal/polyclonal antibodies (Santa Cruz Biotechnology Inc.) for 3 hours, followed by washing with TBST and incubation with antimouse/antirabbit-IgG-HRP conjugate $(1: 40,000)$ for 2 hours. The membranes were incubated with a chemiluminescent substrate (Sigma-Aldrich Co.) and bands were developed using X-ray films. (Kodak, Rochester, NY, USA). The density of the bands was quantified using LabWorks 4.0 Image Analysis software (UVP, Bioimaging Systems, Upland, CA, USA).

\section{Polymerase chain reaction array using real-time quantitative polymerase chain reaction system}

Real time polymerase chain reaction (PCR) technique was used to study mRNA expression in muscle. Total RNA was extracted from muscle homogenates by using Trizol method. The RNA quality and quantity were estimated both by agarose gel electrophoresis and spectrophotometrically. Reverse transcription was performed with SABiosciences' RT2 First Strand Kit (C-03; Qiagen NV, Venlo, the Netherlands). RNA input was $1,000 \mathrm{ng}$ per real time reaction. cDNA template was then run on hypoxia signaling PCR array (Cat No PARN 0032Z) in an ABI one-step plus sequence detection system. PCRs were performed to evaluate the expression of 84 genes using RT2 Prolifer ${ }^{\text {TM }}$ PCR array. Data analysis was performed using RT2 profiler ${ }^{\mathrm{TM}}$ PCR array data analysis version 3.5 (Qiagen NV, Venlo, the Netherlands).

\section{Statistical analysis}

All the experiments were performed two times. Data are presented as mean \pm SD. Data were analyzed using one-way analysis of variance with post hoc Bonferroni analysis using 
standard Statistical Software Package for Social Science (version 16.0; SPSS Inc., Chicago, IL, USA). $P<0.05$ and $P<0.01$ were considered as significant.

\section{Results}

Significant boost in physical performance as measured by running time till exhaustion was observed after EDHB supplementation both in sedentary and in trained rats as compared to their respective controls (1.5 times), indicating reduction in fatigue. Therefore, we assessed the bioenergetics, the potential of EDHB to facilitate the animal to harness energy from cellular processes such as glycolysis, cellular respiration, and other metabolic processes that can lead to production and utilization of energy in forms such as ATP molecules.

\section{Pretreatment with EDHB facilitates glucose uptake and promotes glycolysis}

The rate of glycolysis depends on active glucose uptake. To evaluate the effectiveness of EDHB in favoring glucose uptake, we measured the expression of glucose transporters
GLUT1 and GLUT4 in muscles (Figure 1A). There was a significant increase in the expression of GLUT1 $(37.5 \%$, $P<0.01)$ and GLUT4 $(44.2 \%, P<0.01)$ in the EDHBsupplemented sedentary group as compared to CS, which was further enhanced in drug training group as compared to CT alone. In addition, decrease $(10.5 \%, P<0.05)$ in the level of plasma glucose was observed in training alone group as compared to CS (Figure 1B). EDHB-supplemented sedentary group also had lower glucose $(15 \%, P<0.05)$ as compared to $\mathrm{CS}$, which was maintained when training was given along with EDHB. Further, EDHB supplementation resulted in enhanced activity of key glycolytic enzymes, hexokinase (24.9\%) and PFKm (17.6\%), significantly $(P<0.05)$ in muscle even in sedentary rats as compared to CS group (Figure $1 \mathrm{C}$ and D).

\section{EDHB promotes aerobic metabolism}

Acetyl-CoA is the starting product for the TCA cycle. Elevated levels of acetyl-CoA were observed in EDHBtreated group even without training as compared to CS, which was further enhanced by EDHB along with training
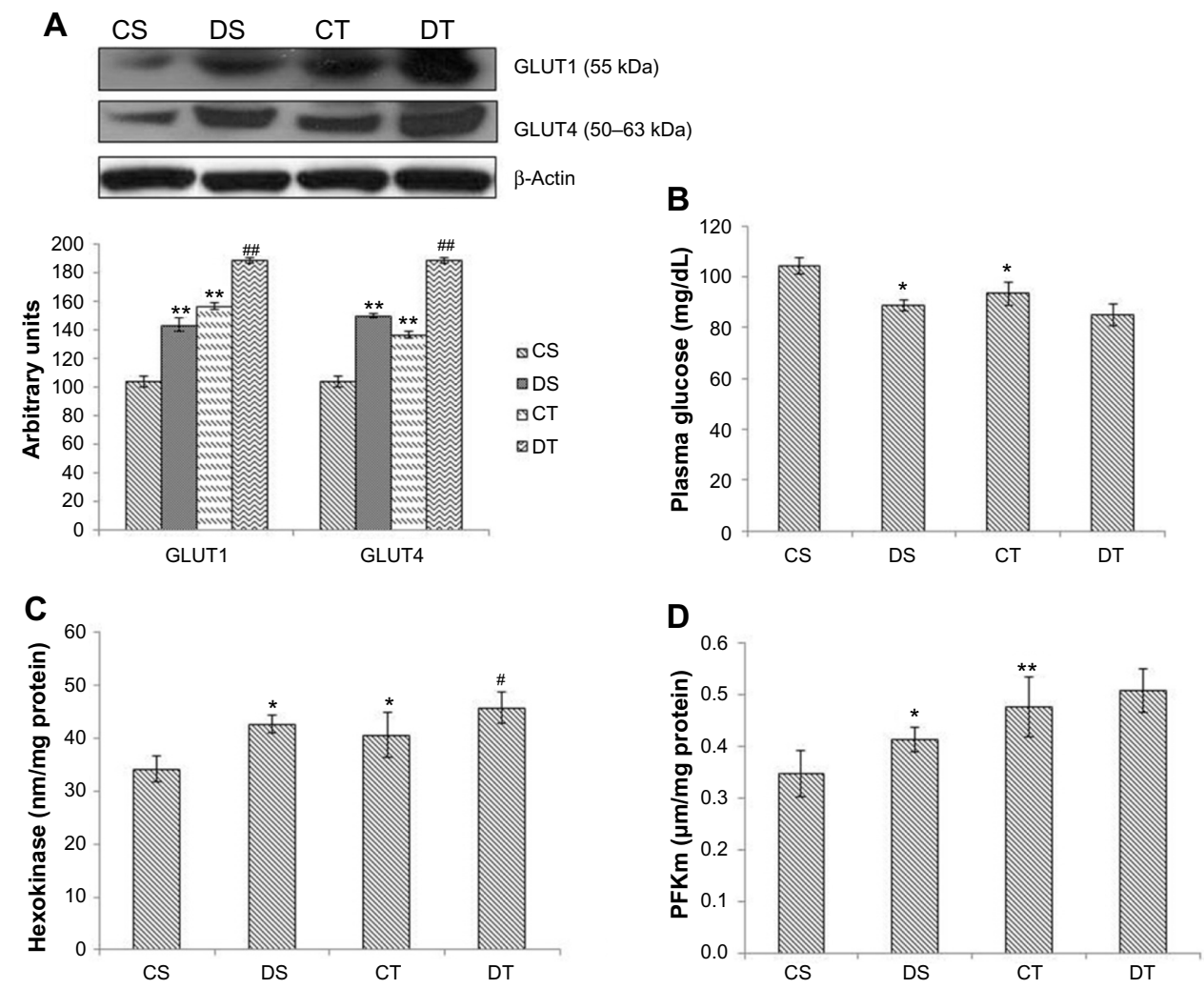

Figure I Effect of EDHB on glycolysis.

Notes: (A) Expression of glucose transporters (GLUTI and GLUT4) was observed in muscle by Western blotting. Representative immunoblots are shown. (B) Glucose levels were estimated in plasma. (C) Hexokinase activity was estimated in rat muscle. (D) Muscle PFK activity. Values are mean \pm SD ( $n=8$ per group). $* * p<0.0$ I vs CS; $* P<0.05$ vs $C S ;{ }^{\#}<0.05$ vs $C T$; and ${ }^{\# P}<0.01$ vs $C T$.

Abbreviations: EDHB, ethyl 3,4-dihydroxybenzoate; PFK, phosphofructokinase; CS, control sedentary; CT, control training; DS, drug sedentary; DT, drug training. 
$(16 \%, P<0.01)$ as compared to training alone group (Figure 2A). This was accompanied by an increase in the activity of citrate synthase and SDH after EDHB supplementation in sedentary and training groups as compared to their respective controls (Figure 2B and C). The increased activity of TCA cycle enzymes resulted in enhanced ATP production (CS vs DS, $49 \%$; CT vs DT, $25 \%$; $P<0.01$ ) as estimated in EDHB-supplemented groups as compared to their respective controls (Figure 2D).

\section{Effect of EDHB on lactate transport}

To investigate the effect of EDHB on lactate transport, plasma level of lactate and expression of lactate transporters were measured. Decreased plasma lactate levels were observed in DS rats $(15 \%, P<0.01)$ as compared to CS group. There was an increase in lactate levels in training alone group, but EDHB supplementation reduced it to a significant level and restored it back to normal. There was a significant decrease $(P<0.01)$ in MCT1 expression in both the training groups as compared to sedentary groups while an increase in MCT4 was observed in EDHB-treated groups as compared to respective controls. EDHB supplementation also resulted in increased activity of LDH in muscle as compared to control groups. These results thus suggest transport of lactate from muscle to plasma after EDHB supplementation (Figure 3).

\section{Effect of EDHB on glycogen and pyruvate in liver and muscle}

Levels of glycogen and pyruvate were estimated in the liver and muscle. The liver is the site of gluconeogenesis and in this process, glucose is synthesized from pyruvate. Since EDHB resulted in a decrease in plasma lactate, we estimated the liver pyruvate levels as lactate is taken up by liver and converted to pyruvate for the synthesis of glucose to maintain the levels of blood glucose and muscle glycogen. Significant elevation in the levels of liver pyruvate (CS vs DS, $147 \%$; CT vs DT, $107 \%, P<0.01$ ) and decrease in muscle pyruvate (CS vs DS, $19 \%$; CT vs DT, $27 \%, P<0.01)$ were observed in EDHBsupplemented rats as compared to respective controls. Levels of glycogen were also found to be enhanced in both liver (CS vs DS, $75 \%$; CT vs DT, $66 \%, P<0.01$ ) and muscle (CS vs DS, 54\%; CT Vs DT, 78\%; $P<0.01$ ) after EDHB supplementation as compared to respective controls (Figure 4).

\section{EDHB supplementation enhances fatty acid oxidation}

Fatty acid oxidation is involved in the generation of 3-C compound acetyl-CoA, which is required to generate ATP during intense muscular activity and for the process of glucose synthesis as in the Cori cycle. A considerable boost in the expression of CPTI was found with EDHB supplementation
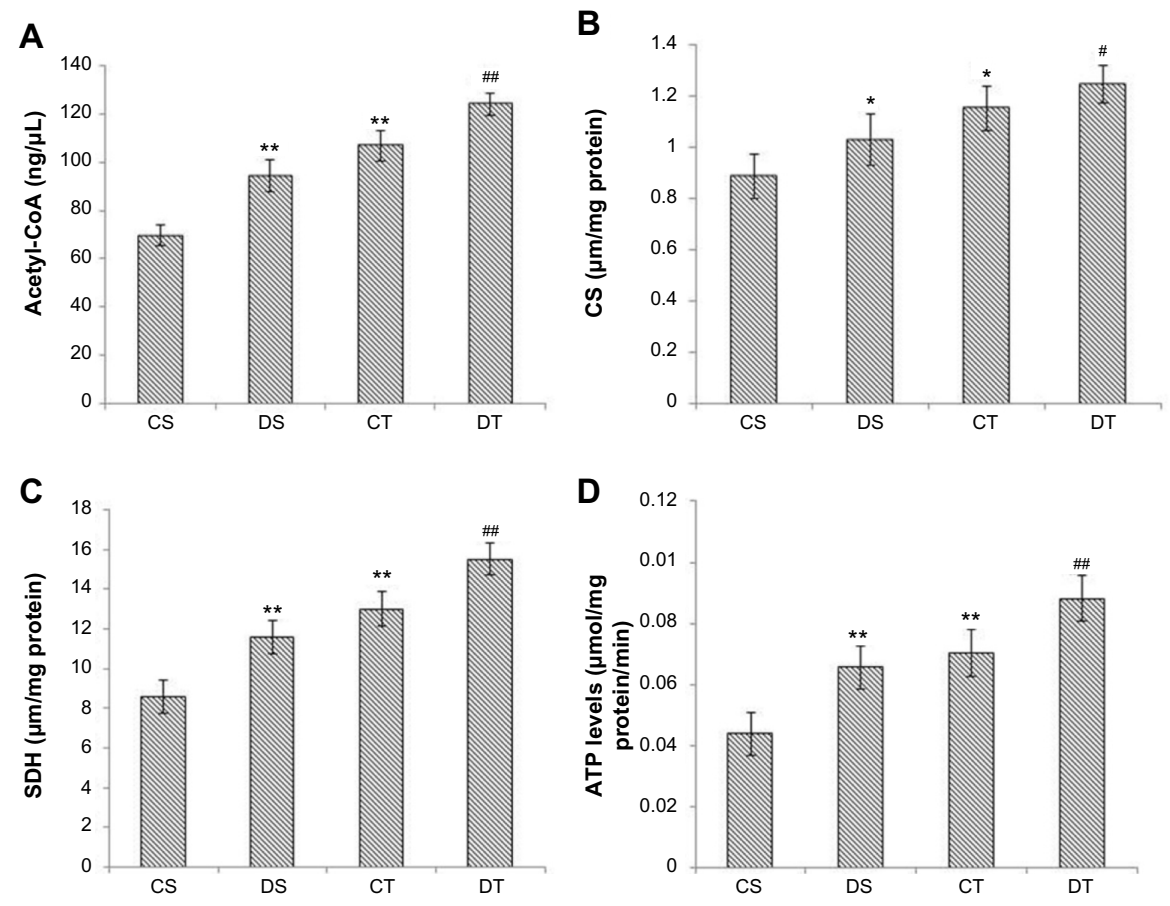

Figure 2 EDHB supplementation promotes energy production by promoting Krebs cycle enzymes activity

Notes: (A) Acetyl-CoA level in muscle. (B) CS activity. (C) SDH activity. (D) ATP level was estimated by phosphorescence. Values are mean \pm SD ( $\mathrm{n}=8$ per group). ${ }^{*} * P<0.0$ l vs $C S ;{ }^{*} P<0.05$ vs $C S ;{ }^{\# P}<0.0$ I vs $C T$; and ${ }^{\#} P<0.05$ vs $C T$.

Abbreviations: EDHB, ethyl 3,4-dihydroxybenzoate; SDH, succinate dehydrogenase; CS, control sedentary; CT, control training; DS, drug sedentary; DT, drug training. 
A
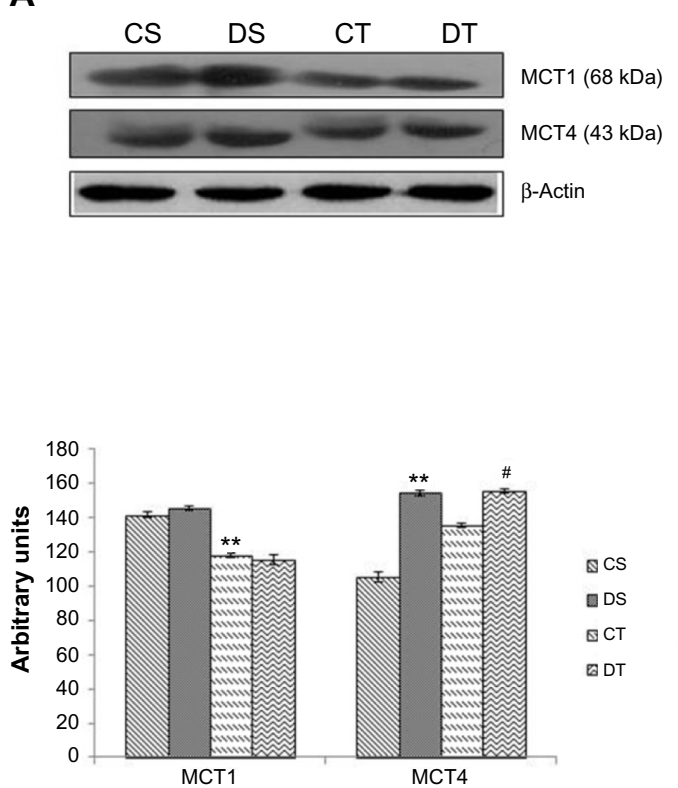
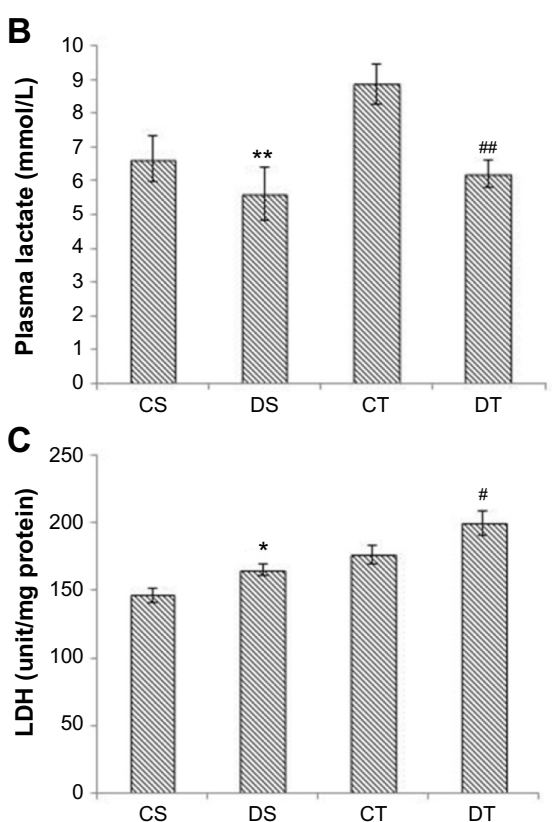

Figure 3 Effect of EDHB supplementation on lactate and lactate transporters.

Notes: (A) Expression of lactate transporters by Western blotting. Representative immunoblots are shown. (B) Lactate level in plasma. (C) LDH activity in rat muscle. Values are mean $\pm S D$ ( $n=8$ per group). ${ }^{* * P}<<0.01$ vs $C S ; * P<0.05$ vs $C S ; \# P<0.01$ vs $C T$; and ${ }^{\#} P<0.05$ vs $C T$.

Abbreviations: EDHB, ethyl 3,4-dihydroxybenzoate; LDH, lactate dehydrogenase; CS, control sedentary; CT, control training; DS, drug sedentary; DT, drug training; MCT, monocarboxylate transporter.

even in sedentary rats as compared to CS, and there was no further change after training. PDK1 remained unchanged with EDHB supplementation in sedentary rats but was increased after training alone and further enhanced when EDHB was given along with training $(20 \%, P<0.01)$. Similarly, augmentation in PDK4 was detected in EDHBsupplemented trained rats as compared to unsupplemented trained controls. PDKs have the ability to switch off pyruvate dehydrogenase complex and thus inhibit the conversion of pyruvate to acetyl-CoA. This increased expression of PDK and CPT thus provides the evidence for activation of fatty acid oxidation (Figure 5).

\section{EDHB supplementation reduces fatigue}

In addition to lactate, increased levels of $\mathrm{Pi}$ also indicate skeletal muscle fatigue. Decreased levels of Pi were observed in EDHB-supplemented rats (11\%, DS vs CS, $P<0.01)$ as compared to CS group. Training along with EDHB supplementation further reduced the levels $(16 \%$, DT vs $\mathrm{CT}, P<0.01)$ as compared to trained control rats,
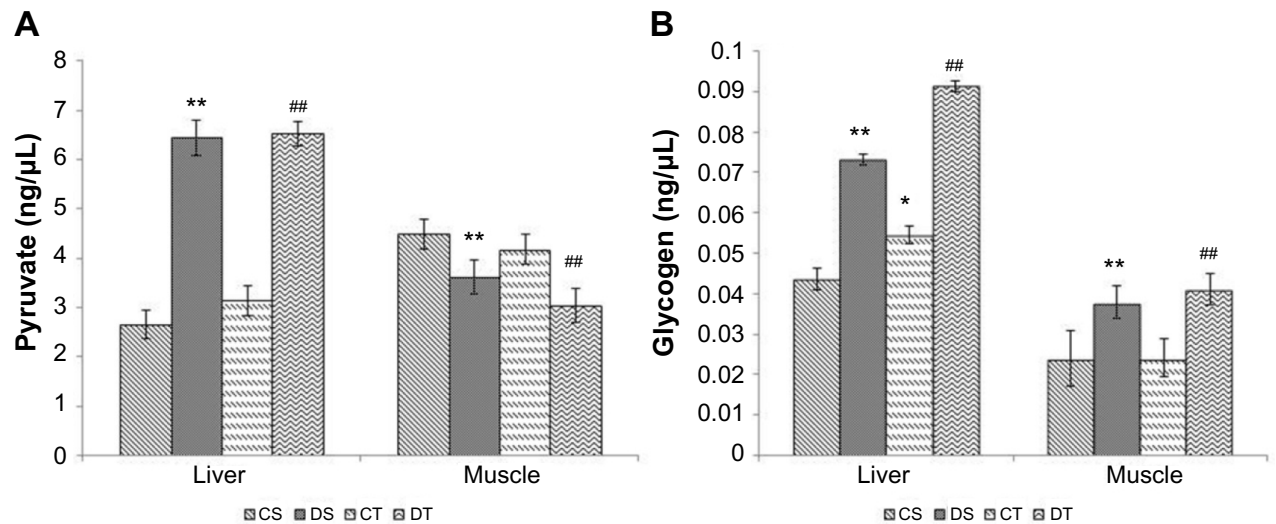

Figure 4 Effect of EDHB supplementation on pyruvate $(\mathbf{A})$ and glycogen $(\mathbf{B})$ in rat liver and muscle.

Notes: Values are mean $\pm S D$ ( $n=8$ per group). ${ }^{* *} P<0.0$ I vs $C S ; * P<0.05$ vs $C S ;{ }^{\#} P<0.0$ I vs $C T$.

Abbreviations: EDHB, ethyl 3,4-dihydroxybenzoate; CS, control sedentary; CT, control training; DS, drug sedentary; DT, drug training. 


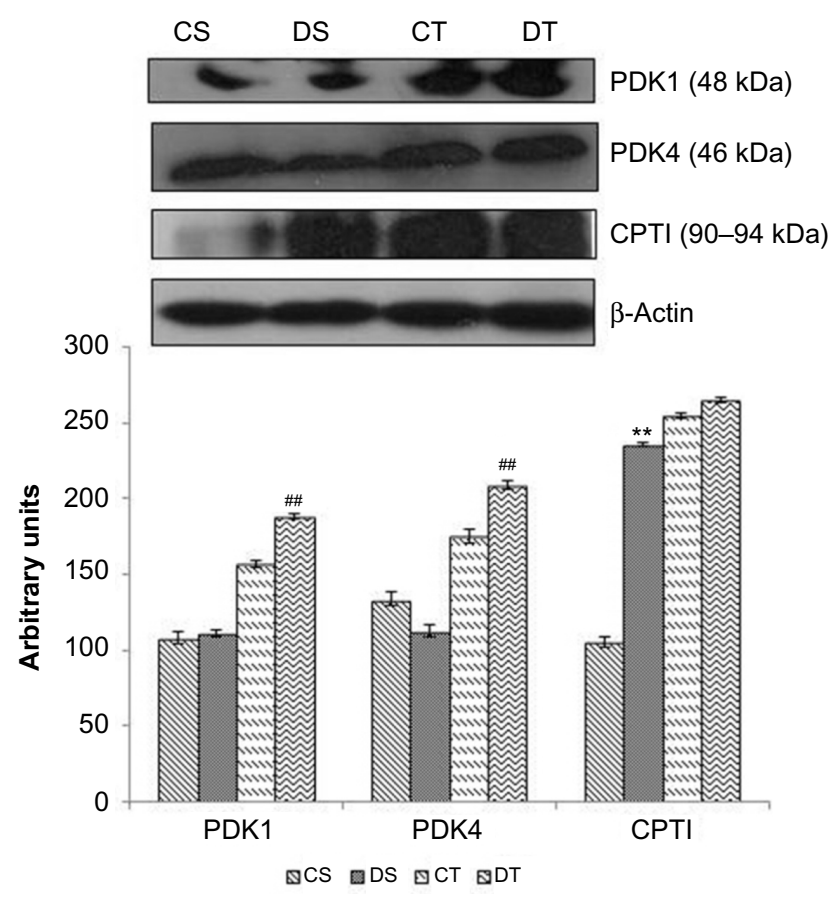

Figure 5 Effect of EDHB supplementation on fatty acid oxidation.

Notes: Expression of proteins involved in fatty acid oxidation was studied by Western blotting in muscle homogenate. Representative immunoblots are shown. Values are mean $\pm S D$ ( $n=8$ per group). ${ }^{* *} P<0.01$ vs $C S ; \# P<0.01$ vs $C T$.

Abbreviations: EDHB, ethyl 3,4-dihydroxybenzoate; CS, control sedentary; CT, control training; DS, drug sedentary; DT, drug training; PDK, pyruvate dehydrogenase kinase; CPTI, carnitine palmitoyltransferase I.

suggesting the protection to the muscles against fatigue (Figure 6).

\section{PCR array analysis}

Hypoxia signaling pathway studies by PCR array revealed upregulation of certain genes by EDHB supplementation,

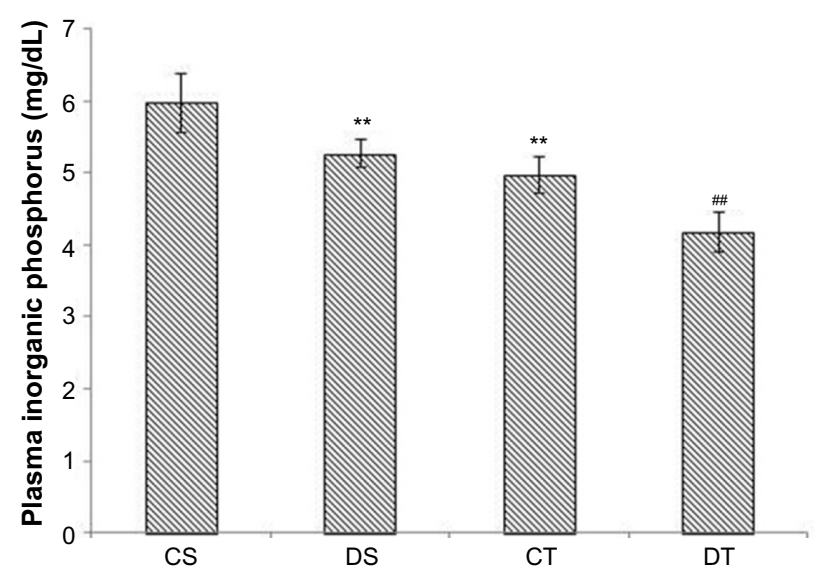

Figure 6 EDHB supplementation reduces fatigue.

Notes: Level of inorganic phosphate in plasma. Values are mean \pm SD $(n=8$ per group). ${ }^{* * P}<0.0$ I vs $C S ; \# P<0.01$ vs $C T$.

Abbreviations: EDHB, ethyl 3,4-dihydroxybenzoate; CS, control sedentary; CT, control training; DS, drug sedentary; DT, drug training. which helps the skeletal muscle to adapt better under stress. We observed upregulation in the expression of various genes in EDHB-supplemented groups as compared to sedentary control (Figure 7 and Table 1).

\section{Discussion}

Endurance exercise such as prolonged running induces an increase in muscle respiratory capacity. ${ }^{22}$ This adaptation includes an increase in components of the mitochondrial respiratory chain, ATP synthase, enzymes of citrate cycle, and those involved in fatty acid oxidation. ${ }^{23,24}$ This enhancement of muscle respiratory capacity induced by training plays a major role in the improvement of exercise performance and endurance. ${ }^{25}$ In this study, preconditioning with EDHB resulted in augmentation of bioenergetics even without training, thus facilitating improvement in physical performance or reduction in fatigue, which was measured by running time till exhaustion in rats.

The mitochondrion is often referred to as the powerhouse of the cell. It contains all the machinery needed to provide the cell and its components with energy to carry out cellular processes. The matrix of the mitochondrion is where the TCA cycle occurs, in which pyruvate (oxidized from glucose in glycolysis) is converted into acetyl-CoA and then fed into the pathway to be oxidized to $\mathrm{CO}_{2}$ and its energy conserved. Glycolysis is the metabolic process that converts glucose into lactate and yield high-energy compounds such as ATP and NADH for body functioning. Availability of glucose is one important aspect of glycolysis. For the process of glycolysis, glucose can be taken up either from glycogen stored in the muscle or from blood with the help of glucose transporters such as GLUT1 and GLUT4. ${ }^{26,27}$ Both blood glucose and muscle glycogen are essential for prolonged strenuous exercise, and exhaustion can result either from the development of hypoglycemia or from depletion of muscle glycogen. To support active glucose uptake, we studied the expression of glucose transporters GLUT1 and GLUT4 in muscle, which was upregulated, thereby enhancing glucose uptake. GLUT4 permits the facilitated diffusion of circulating glucose into muscle, while GLUT1 facilitates the transport of glucose across blood-tissue barriers. The interaction between the two transporters thus helps in active glucose uptake. ${ }^{28,29}$

In this study, there was an increment in the activity of two important glycolytic enzymes hexokinase and Phosphofructokinase (muscle) that are found in many organisms, including bacteria, plants, and mammals, and play an important role in glucose metabolism. Since muscle lacks glucose6-phosphatase, any glucose that enters the muscle will be 


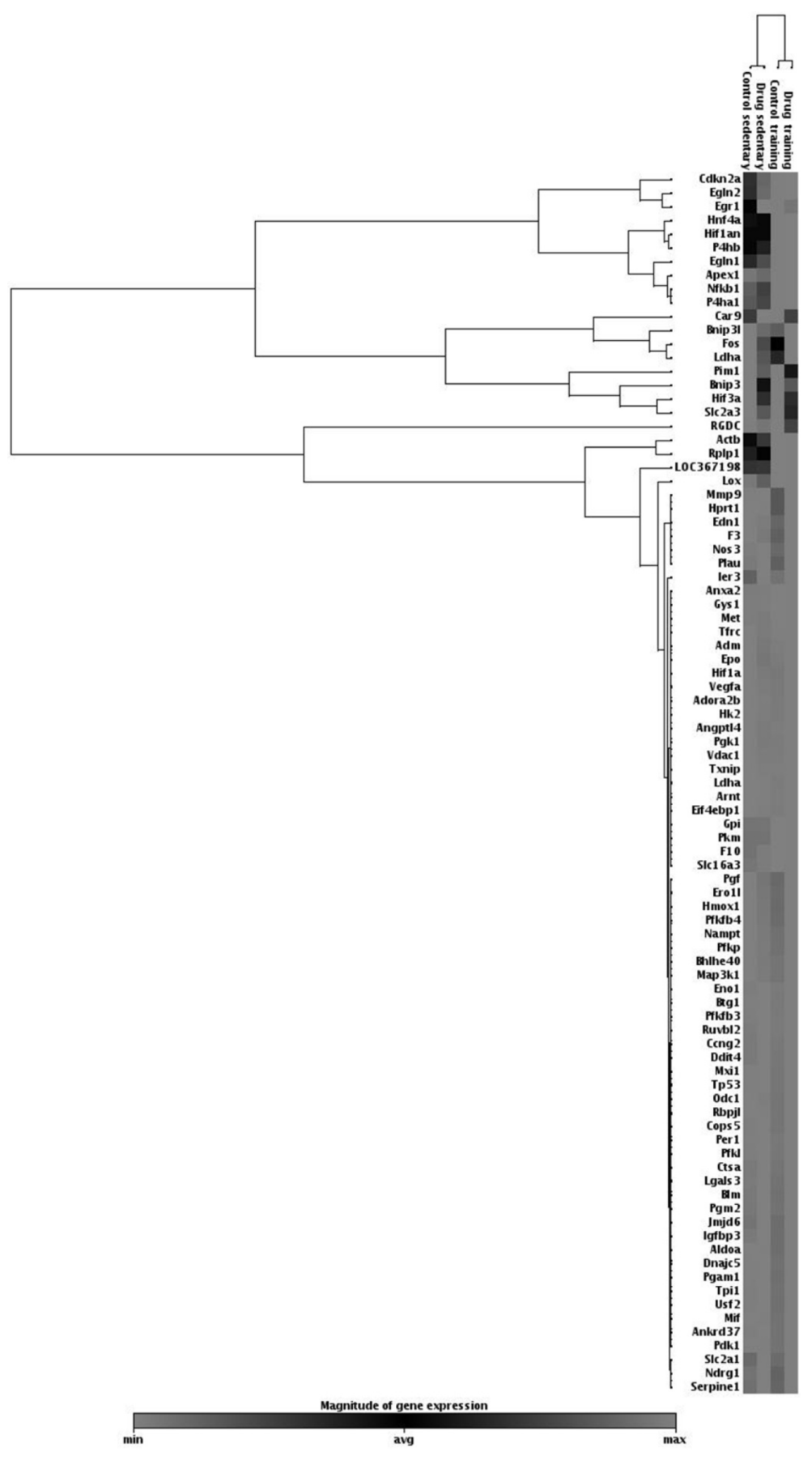

Figure 7 Scatter plot of hypoxia signaling PCR array studies for the metabolic genes listed.

Abbreviations: PCR, polymerase chain reaction; min, minimum; avg, average; max, maximum.

phosphorylated by hexokinase and remain in the muscle to be either be metabolized immediately or stored as glycogen for later use. ${ }^{25} \mathrm{PFK}$ has three major isoforms in mammals: PFK-M (muscle), PFK-L (liver), and PFK-P (platelet). In humans, PFK deficiency causes glycogen storage disease, also called Tarui's disease, which is characterized by exerciseinduced muscle weakness and cramps. In the process of glycolysis, hexokinase catalyzes the first reaction of formation of glucose-6-phosphate (G6P) by transferring a phosphoryl group from ATP, while PFK catalyzes the irreversible transfer of phosphate from ATP to fructose-6-phosphate. ${ }^{29,30}$

The fate of pyruvate in glycolysis depends on the availability of oxygen. Under aerobic conditions, pyruvate is converted to acetyl-CoA by the enzyme pyruvate dehydrogenase, which thus enters the Krebs cycle where a large number of ATP molecules are generated, whereas under anaerobic 
Table I PCR array results for metabolic genes

\begin{tabular}{|c|c|c|c|c|c|}
\hline \multicolumn{6}{|c|}{ Fold change in comparison to control sedentary group } \\
\hline Genes & Full name & CS & DS & CT & DT \\
\hline$\overline{A l d o a}$ & Aldolase $\mathrm{A}$, fructose-bisphosphate & $\mathrm{I}$ & 1.0484 & 2.1097 & 17.6028 \\
\hline Enol & Enolase I, (alpha) & 1 & 0.9075 & 1.1488 & $|2.48| 1$ \\
\hline$H K 2$ & Hexokinase 2 & I & 1.168 & 1.4001 & 20.1989 \\
\hline Ldha & Lactate dehydrogenase $\mathrm{A}$ & 1 & 1.0359 & 1.1639 & 12.2476 \\
\hline$P d k l$ & Pyruvate dehydrogenase kinase, isozyme I & I & 1.0007 & 1.8652 & 18.9609 \\
\hline Pfkfb3 & 6-Phosphofructo-2-kinase/fructose-2,6-biphosphatase 3 & 1 & 0.8635 & 1.5057 & 29.5799 \\
\hline Pfkfb4 & 6-Phosphofructo-2-kinase/fructose-2,6-biphosphatase 4 & 1 & 1.5724 & 2.7437 & 23.5252 \\
\hline$P f k l$ & Phosphofructokinase, liver & I & 0.8826 & 1.5061 & 18.9822 \\
\hline Pgam I & Phosphoglycerate mutase I & 1 & 0.9785 & 1.9367 & 16.2063 \\
\hline Tpi & Triosephosphate isomerase I & I & 0.8993 & 1.6804 & 15.0775 \\
\hline Pgkl & Phosphoglycerate kinase I & I & 1.1485 & 1.138 & 8.5745 \\
\hline Pgm2 & Phosphoglucomutase 2 & 1 & 0.691 & 1.2759 & 11.4361 \\
\hline$P k m$ & Pyruvate kinase, muscle & 1 & 0.9843 & 0.6885 & 6.5171 \\
\hline Slcl6a3 & $\begin{array}{l}\text { Solute carrier family } 16 \text {, member } 3 \text { (monocarboxylic acid } \\
\text { transporter 4) }\end{array}$ & 1 & 0.8398 & 0.7585 & 6.9987 \\
\hline Slc2al & $\begin{array}{l}\text { Solute carrier family } 2 \text { (facilitated glucose transporter), } \\
\text { member I }\end{array}$ & 1 & 0.5671 & 0.975 & 5.7985 \\
\hline Gpi & Glucose phosphate isomerase & 1 & 0.997 & 0.7527 & 6.4342 \\
\hline Gys I & Glycogen synthase I, muscle & I & 0.9625 & 0.9143 & 9.4455 \\
\hline
\end{tabular}

Abbreviations: PCR, polymerase chain reaction; CS, control sedentary; DS, drug sedentary; CT, control training; DT, drug training.

conditions, pyruvate undergoes either lactic acid fermentation or alcohol fermentation with a low yield of ATP. In this study, an increase in muscle LDH activity and a decrease in muscle pyruvate levels were observed in EDHB-supplemented rats, suggesting conversion of pyruvate to lactate. Further, reduced expression of MCT1 and increase in MCT4 after EDHB supplementation suggest transport of lactate from muscle to blood. Decrease in plasma lactate level by EDHB supplementation might be due to enhanced transport of lactate from blood to liver and conversion to pyruvate as there was a rise in liver pyruvate levels. Membrane-bound MCTs facilitate cell-to-cell exchange of lactate, and two isoforms MCT1 and MCT4 are the most active forms in skeletal muscles. MCT1 channels the lactate from blood to skeletal muscle, and MCT4 is involved in cellular lactate extrusion. ${ }^{31,32}$ Here, we observed a decrease in MCT1 expression in both the training groups as compared to sedentary groups, while an increase in MCT4 was observed in DS and training group as compared to respective controls.

As glycolytic pathways are stressed in skeletal muscle (ie, high-intensity exercise), pyruvate accumulates in the absence of oxygen, thus favoring lactate formation. Lactate is transported from working skeletal muscle into the bloodstream and is taken up by nonworking skeletal muscle and the liver. Nonworking skeletal muscle and liver convert lactate back into pyruvate, which is either metabolized via the TCA cycle or the liver converts the pyruvate into glucose (or glycogen) through gluconeogenesis and releases the glucose into the bloodstream. ${ }^{33}$ The working skeletal muscle will use the circulating blood glucose for energy production. ${ }^{34,35}$ Pyruvate is utilized in gluconeogenesis, leading to increased glucose and subsequently glycogen levels in the liver as observed in EDHB-supplemented rats, which were further increased by training along with EDHB supplementation as compared to respective controls.

The adaptations induced by endurance exercise training result in a marked sparing of carbohydrate during exercise, with an increased proportion of the energy being provided by fat oxidation. PDK1 and PDK4 suppress pyruvate dehydrogenase complex and thus conversion of pyruvate to acetyl-CoA, which results in the reduction of ATP production by shifting pyruvate from mitochondria. ${ }^{36,37} \mathrm{We}$ observed upregulation of PDK1 and PDK4 expressions in both the training groups, the increase being more when EDHB was given along with training, pointing toward a decrease in acetyl-CoA. Interestingly, enhanced level of acetyl-CoA was observed in this study in EDHB-supplemented groups, which could be due to enhanced fatty acid oxidation as there was an increase in the expression of CPTI after EDHB supplementation even without training, which helps in fatty acid oxidation.

Transport of fatty acid is based on carnitine-dependent transport system. ${ }^{38} \mathrm{~L}-\mathrm{Carnitine}$ is the compound responsible for the transport of long-chain fats (as long-chain acyl-CoA) across the otherwise impermeable inner mitochondrial membrane. CPTI is the rate-limiting enzyme of the fatty acid oxidation pathway. ${ }^{39}$ CPTI loads free carnitine with 


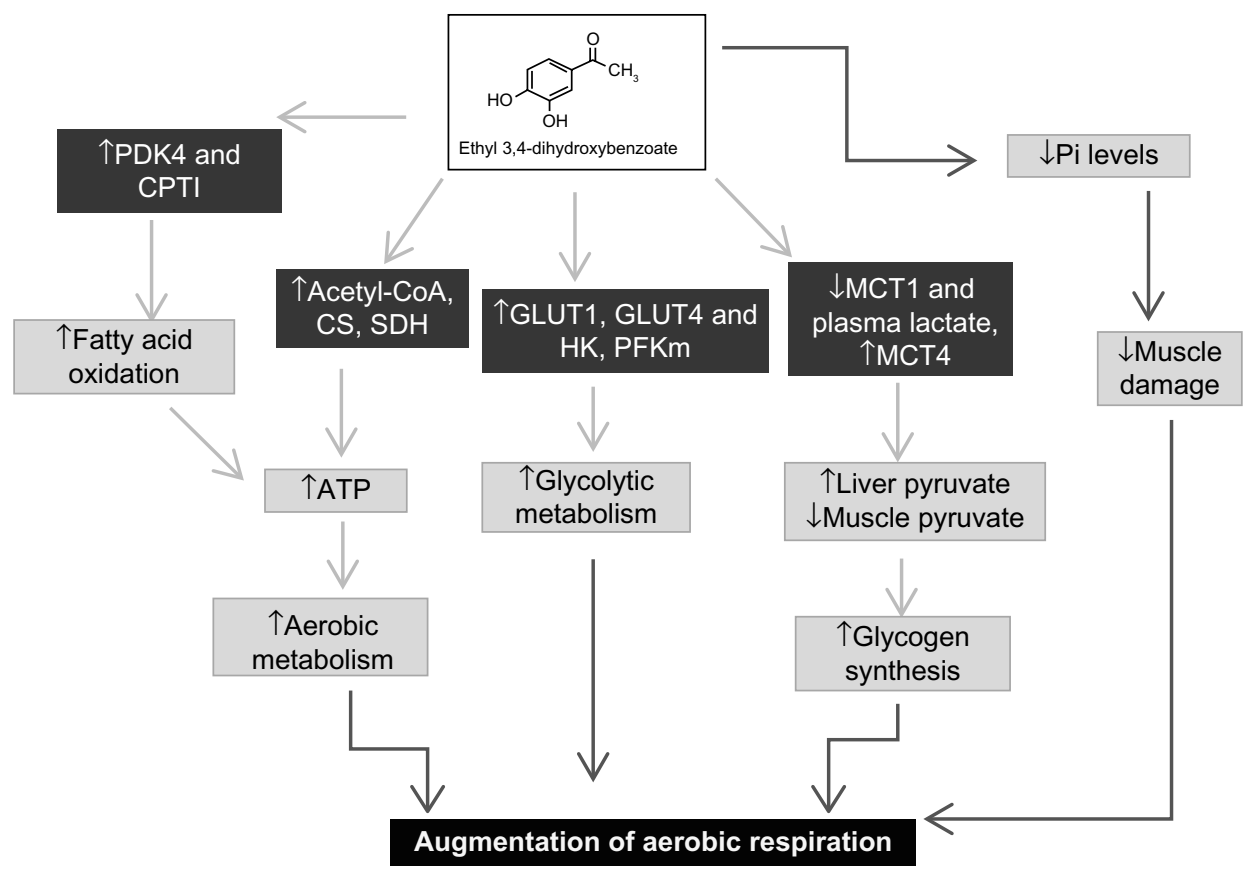

Figure 8 Putative mechanism of EDHB in augmentation of physical performance.

Notes: $\uparrow$ upregulation by EDHB supplementation and $\downarrow$ downregulation by EDHB.

Abbreviations: EDHB, ethyl 3,4-dihydroxybenzoate; PDK, pyruvate dehydrogenase kinase; CPTI, carnitine palmitoyltransferase I; Pi, inorganic phosphate; SDH, succinate dehydrogenase; CS, control sedentary; HK, hexokinase; $\mathrm{MCT}$, monocarboxylate transporter.

the long-chain fatty acids. Once this has taken place, the long-chain acylcarnitine compound is transported across the mitochondrial membrane. Fatty acids are broken down into acetyl-CoA in a four-step $\beta$-oxidation process. AcetylCoA produced by the metabolism of fats is also oxidized within the mitochondria to form $\mathrm{CO}_{2}$ and $\mathrm{H}_{2} \mathrm{O} .{ }^{40}$ Thus, the enhanced level of acetyl-CoA resulted in augmentation of the Krebs cycle, which was evident by the increase in SDH and citrate synthase, resulting in the production of high levels of ATP in EDHB-treated groups as compared to controls. This increase in both the enzymes leads to increase in Krebs cycle activity, thus augmenting production of energy in the form of ATP, which is considered as the energy currency of life. In accordance, we observed a considerable boost in levels of ATP in EDHB-treated rats as compared to respective controls. PCR studies further revealed active upregulation of genes involved in metabolism, viz, aldolase A, which is a key enzyme in glycolysis and in the reverse pathway gluconeogenesis; fructose-bisphosphatase one of the important enzymes of gluconeogenesis; enolase 1, (alpha) a glycolytic enzyme that plays a role in glycolysis and gluconeogenesis; hexokinase 2, LDH A, pyruvate dehydrogenase kinase 1, and 6-phosphofructo-2-kinase-1, 2, 3 (PFK 1, 2, 3); liver phosphoglycerate mutase 1 , triosephosphate isomerase 1 , phosphoglycerate kinase 1, phosphoglucomutase 2, pyruvate kinase, muscle, solute carrier family 16, member 3 (MCT4), solute carrier family 2 (GLUT1), glucose phosphate isomerase, and muscle glycogen synthase 1 (Table 1). All these enzymes play important roles in metabolic cycles, and upregulation of these enzymes suggests an active regulation of various metabolic pathways by EDHB supplementation, thus facilitating cellular aerobic respiration.

Activation of the metabolic pathways that produce ATP also results in increased muscle and plasma levels of numerous metabolic by-products such as Pi that potentially contribute to fatigue during exercise. During exercise, substantial decline in force and/or power output due to fatigue has gained considerable attention. Anaerobic metabolism in skeletal muscle also involves hydrolysis of creatine phosphate to creatine and Pi. Creatine has little effect on contractile function, whereas there are several mechanisms by which increased Pi may depress contractile function. ${ }^{41}$ In our study, decrease in the level of Pi suggests protection against fatigue in EDHB-supplemented groups.

Supplementation of EDHB thus preconditions the body to face the challenges of subsequent exercise training by improving bioenergetics and metabolic status of the rats, resulting in enhanced physical performance through the escalation of aerobic respiration and ATP production in the skeletal muscles (Figure 8). 


\section{Acknowledgments}

This research is supported by financial assistance from the Defense Research and Development Organisation (DRDO), Government of India. The authors are thankful to the Director, Dr SB Singh, DIPAS, DRDO, India, for providing all the support and facilities for conducting this research. The authors also wish to thank Mr Bhagwat Singh for providing animals for research work.

\section{Author contributions}

All authors contributed toward data analysis, drafting and critically revising the paper and agree to be accountable for all aspects of the work.

\section{Disclosure}

Ms Charu Nimker and Mr Deependra Pratap Singh are recipients of Senior Research fellowship from the Council of Scientific and Industrial Research (CSIR), Government of India. The authors report no other conflicts of interest in this work.

\section{References}

1. Matsakas A, Patel K. Skeletal muscle fibre plasticity in response to selected environmental and physiological stimuli. Histol Histopathol. 2009;24(5):611.

2. Hoppeler H, Vogt M. Muscle tissue adaptations to hypoxia. J Exp Biol. 2001;204(18):3133-3139.

3. Poole DB, Mathieu-costello O. Relationship between fiber capillarization and mitochondrial volume density in control and trained rat soleus and plantaris muscles. Microcirculation. 1996;3(2):175-186.

4. Egan B, Zierath JR. Exercise metabolism and the molecular regulation of skeletal muscle adaptation. Cell Metab. 2013;17(2):162-184.

5. Holloszy JO, Booth FW. Biochemical adaptations to endurance exercise in muscle. Annu Rev Physiol. 1976;38(1):273-291.

6. Pilegaard H, Ordway GA, Saltin B, Neufer PD. Transcriptional regulation of gene expression in human skeletal muscle during recovery from exercise. Am J Physiol-Endocrinol Metabol. 2000;279(4): E806-E814.

7. Baar K, Wende AR, Jones TE, et al. Adaptations of skeletal muscle to exercise: rapid increase in the transcriptional coactivator PGC-1. FASEB J. 2002;16(14):1879-1886.

8. Akimoto T, Pohnert SC, Li P, et al. Exercise stimulates Pgc-1 $\alpha$ transcription in skeletal muscle through activation of the p38 MAPK pathway. J Biol Chem. 2005;280(20):19587-19593.

9. Lindholm ME, Rundqvist H. Skeletal muscle hypoxia-inducible factor-1 and exercise. Exp Physiol. 2016;101(1):28-32.

10. Lundby C. Skeletal muscle adaptations to endurance training: is tissue hypoxia the main signal? Exp Physiol. 2016;101(1):15-16.

11. Zoll J, Ponsot E, Dufour S, et al. Exercise training in normobaric hypoxia in endurance runners. III. Muscular adjustments of selected gene transcripts. J Appl Physiol. 2006;100(4):1258-1266.

12. Nimker C, Kaur G, Revo A, Chaudhary P, Bansal A. Ethyl 3,4-dihydroxy benzoate, a unique preconditioning agent for alleviating hypoxiamediated oxidative damage in L6 myoblasts cells. J Physiol Sci. 2015;65(1):77-87.

13. Nimker C, Singh D, Kaur G, Saxena S, Bansal A. Protective efficacy of ethyl 3, 4-dihydroxy benzoate against exercise induced damages: putative role in improving physical performance. Int J Pharma Sci Res. 2015;6(6):2423-2436.
14. Singh DP, Nimker C, Paliwal P, Bansal A. Ethyl 3,4-dihydroxybenzoate (EDHB): a prolyl hydroxylase inhibitor attenuates acute hypobaric hypoxia mediated vascular leakage in brain. J Physiol Sci. Epub 2015 Dec 9.

15. Bejma J, Ji LL. Aging and acute exercise enhance free radical generation in rat skeletal muscle. J Appl Physiol (1985). 1999;87(1):465-470.

16. Gonchar O. Muscle fiber specific antioxidative system adaptation to swim training in rats: influence of intermittent hypoxia. J Sports Sci Med. 2005;4(2):160-169.

17. Srere PA. Citryl-CoA and the citrate condensing enzyme. Biochim Biophys Acta. 1963;77:693-696.

18. Veeger C, DerVartanian D, Zeylemaker W. Succinate dehydrogenase:[EC 1.3. 99.1 Succinate:(acceptor) oxidoreductase]. Methods Enzymol. 1969;13:81-90.

19. Holroyde MJ, Trayer IP. Purification and properties of rat skeletal muscle hexokinase. FEBS Lett. 1976;62(2):215-219.

20. Ling K, Paetkau V, Marcus F, Lardy HA. Phosphofructokinase: I. Skeletal muscle. Methods Enzymol. 1966;9:425-429.

21. Goldenberg H, Fernandez A. Simplified method for the estimation of inorganic phosphorus in body fluids. Clin Chem. 1966;12(12): $871-882$.

22. Booth FW, Baldwin KM. Muscle plasticity: energy demand and supply processes. Compr Physiol. 1996;(Suppl 29):1075-1123.

23. Oscai LB, Holloszy JO. Biochemical adaptations in muscle II. Response of mitochondrial adenosine triphosphatase, creatine phosphokinase, and adenylate kinase activities in skeletal muscle to exercise. J Biol Chem. 1971;246(22):6968-6972.

24. Chi M, Hintz C, Coyle E, et al. Effects of detraining on enzymes of energy metabolism in individual human muscle fibers. Am J Physiol. 1983;244(3):C276-C287.

25. Holloszy JO, Coyle EF. Adaptations of skeletal muscle to endurance exercise and their metabolic consequences. J Appl Physiol. 1984; 56(4):831-838.

26. Semenza GL, Roth PH, Fang HM, Wang GL. Transcriptional regulation of genes encoding glycolytic enzymes by hypoxia-inducible factor 1 . J Biol Chem. 1994;269(38):23757-23763.

27. Semenza GL. Oxygen sensing, homeostasis, and disease. N Engl J Med. 2011;365(6):537-547.

28. Zhao FQ, Keating AF. Functional properties and genomics of glucose transporters. Curr Genomics. 2007;8(2):113-128.

29. Lenzen S. A fresh view of glycolysis and glucokinase regulation: history and current status. J Biol Chem. 2014;289(18):12189-12194.

30. El-Gharbawy A, Koeberl D. Basic biochemical pathway, clinical presentation, diagnosis, and treatment. Inborn Errors Metabol. 2014;64:119-133.

31. Morris ME, Felmlee MA. Overview of the proton-coupled MCT (SLC16A) family of transporters: characterization, function and role in the transport of the drug of abuse $\gamma$-hydroxybutyric acid. AAPS J. 2008;10(2):311-321.

32. Sepponen K, Koho N, Puolanne E, Ruusunen M, Pösö A. Distribution of monocarboxylate transporter isoforms MCT1, MCT2 and MCT4 in porcine muscles. Acta Physiol Scand. 2003;177(1):79-86.

33. Facey A, Irving R, Dilworth L. Overview of lactate metabolism and the implications for athletes. Am J Sports Sci Med. 2013;1(3):42-46.

34. Gladden LB. Lactate metabolism: a new paradigm for the third millennium. J Physiol. 2004;558(1):5-30.

35. Wirtz N, Wahl P, Kleinöder H, Mester J. Lactate kinetics during multiple set resistance exercise. J Sports Sci Med. 2014;13(1):73-77.

36. Kim JW, Tchernyshyov I, Semenza GL, Dang CV. HIF-1-mediated expression of pyruvate dehydrogenase kinase: a metabolic switch required for cellular adaptation to hypoxia. Cell Metab. 2006;3(3): $177-185$.

37. Papandreou I, Cairns RA, Fontana L, Lim AL, Denko NC. HIF-1 mediates adaptation to hypoxia by actively downregulating mitochondrial oxygen consumption. Cell Metab. 2006;3(3):187-197.

38. Kerner J, Hoppel C. Fatty acid import into mitochondria. Biochim Biophys Acta. 2000;1486(1):1-17. 
39. Stephens FB, Constantin-Teodosiu D, Greenhaff PL. New insights concerning the role of carnitine in the regulation of fuel metabolism in skeletal muscle. J Physiol. 2007;581(2):431-444.

40. Green JH. Energy metabolism. In: The Autonomic Nervous System and Exercise. New York: Springer US; 1990:72-103.
41. Westerblad H, Bruton JD, Allen DG, Lännergren J. Functional significance of $\mathrm{Ca}^{2+}$ in long-lasting fatigue of skeletal muscle. Eur J Appl Physiol. 2000;83(2-3):166-174.

Hypoxia

Dovepress

\section{Publish your work in this journal}

Hypoxia is an international, peer-reviewed, open access journal that aims to improve understanding of the biological response to hypoxia. The journal will publish original research articles, reviews, methodological advances, clinical studies, and expert opinions that identify developments in the regulation of the physiological and pathological responses to

Submit your manuscript here: http://www.dovepress.com/hypoxia-journal

hypoxia and in the therapeutic targeting of hypoxia-responsive pathways. The manuscript management system is completely online and includes a very quick and fair peer-review system, which is all easy to use. Visit http://www.dovepress.com/testimonials.php to read real quotes from published authors. 